\title{
InfoScale 2008 - The Third International ICST Conference on Scalable Information Systems
}

\author{
Ronny Lempel, Raffaele Perego, Fabrizio Silvestri
}

Scalability is the key word for today's modern software architectures. As data and knowledge volume keep increasing while global means for information dissemination continue to diversify, new methods, modeling paradigms, and structures are needed to efficiently support the mounting scalability requirements.

In the last few years we have seen the proliferation of the use of heterogeneous distributed systems, ranging from simple Networks of Workstations, to highly complex Distributed Computing systems. Such computational paradigms have been preferred due to their reduced costs and inherent scalability, but their efficient usage poses many challenges in terms of information access, storage and retrieval as well as in terms of algorithms for efficiently managing, transferring, and analyzing huge volume of data and knowledge. Furthermore, with the advent of emerging computing architectures - e.g. SMTs, GPUs, Multicores, etc. the importance of designing techniques explicitly targeting these systems, is becoming more and more important.

We would like to thank ICST for their effort and continuous help with the "material" organization of the conference, as well as the "spiritual" support they provided in the last few weeks. In particular, we thank Dorothy Bany for her valuable continuous contribution. Special thanks to our Program Committee Chairs - Ricardo Baeza-Yates, Abdur Chowdhury, and Cheng-Zhong Xu - for their many hours of work on the conference. We would like to thank our sponsors, Yahoo! Research and the Italian National Research Council, for their generous financial contributions to the conference. Finally, we are honored to have as our honorary chair Andrei Broder from Yahoo! Research. For us, it has been a delightful pleasure to working with all of you! Last but not least, we would like to express all our gratitude to Imrich Chlamtac and Ophir Frieder for having invited us to chair the organization of the third conference in the InfoScale series.

The three keynotes that are scheduled for this year's conference are given by three well-known scientists in their respective fields: Giorgio Ventre, Ant Rowstron, and Ricardo Baeza-Yates. They will light up our work days with enlightening ${ }^{1}$ talks on trailblazing topics.

On behalf of the Institute for Computer Science, Social Informatics and Telecommunications Engineering (ICST), we are delighted to welcome you to the beautiful city of Vico Equense in Naples. We hope you will enjoy both the conference and the surroundings - the breathless sightseeing and the marvelous natural scenery this city offers.

Ronny, Raffaele \& Fabrizio

${ }^{1}$ Needless to say again: pun intended!

INFOSCALE 2008, June 4-6, Vico Equense, Italy

Copyright (C) 2008 978-963-9799-28-8

DOI 10.4108/ICST.INFOSCALE2008.3575 